\title{
Classification of different erythrocyte cells by using bioimpedance surface acoustic wave and their sedimentation rates
}

\author{
Mohamed A. A. Eldosoky \\ Department of Biomedical Engineering, Helwan University, Egypt \\ E-mail any correspondence to: hm1_eldosoky@hotmail.com, mohamed_eldesouky@h-eng.helwan.edu.eg
}

\begin{abstract}
Classification of the RBCs with their shapes, volumes and volume fractions is an important indicator for the normality of the healthy body. RBCs in the plasma are simulated electrically as a conductor solution with insulated particles moving through the plasma. Consequently, the impedance of the plasma-RBCs is proportional to the number and the volume of the RBCs within the plasma. This paper presents a new proposed method for studying the characteristics of the RBCs by using a surface acoustic wave sensor. Because of the free motion of the RBCs during the test of the erythrocytes sedimentation rate, the concentration of the RBCs varies from one layer to another. Consequently, the output waveform of the surface acoustic wave sensor changes from one time to another related to the behavior of the RBCs. This method shows its ability to classify not only the volume fraction and volumes of the RBCs, but also the different types of the RBCs.
\end{abstract}

Keywords: Bioimpedance, erythrocytes sedimentation rate, RBCs, surface acoustic waves.

\section{Introduction}

Blood is the vital liquid that holds the secrets of the whole body. The major part of it is called RBCs. The rest of it is WBCs and platelets. All these components float in liquid called plasma. Any variation in the shape or the volume or the number of them in the blood is inherent with not only the blood diseases but also the human organs diseases.

$\mathrm{RBCs}$ in the plasma can be represented electrically as insulating particles (RBCs) in a conducting liquid (plasma).

Due to the difference in the electrical characteristics between the RBCs and the plasma, any variation in the characteristics of the RBCs (blood cell concentrations, their volumes, and their modalities) will have an image on the related electrical impedance of the RBCs-plasma [1].

Many mathematical models of the RBCs-plasma solution had been proposed for determining the electrical characteristics of this solution at different cases of RBCs modalities, concentrations, and volumes [2]. Besides that, the electrical polarization dependent shape had been studied in [3].

Another technique for determining the electrical impedance of the solution has been applied as a tool for determining the solutions' concentration by using signals at three different frequencies from three different electrodes [4].

According to the significant difference in the electrical characteristics between the RBCs and the plasma, bioimpedance analysis has an apparent role in estimating the characteristics of the RBCs within the plasma and furthermore diseases related to the RBCs' characteristics [5-8].

The abnormality of the RBCs may relate to the number, the shape, or the volume of the RBCs. Microcytes and macrocytes are two cases of abnormal RBCs. Their characteristics have been estimated as compared to the spherical cases [9].

This paper presents a proposed model as a diagnostic tool for the RBCs including their electrical characteristics. This method relies on studying the impedance response of the RBCs-plasma solution during the sedimentation process of the red blood cells by using surface acoustic wave (SAW). 
Surface acoustic wave (SAW) is with high sensitivity responsible for detecting the variation in the electrical characteristics of the solution with the passage of the time.

This method can be modified as a classification tool for determining the erythrocytes sedimentation rate (ESR) and classifying the modalities, the volumes, and the volume fractions of the RBCs. This model has proved the SAW's ability for classifying the variation of the RBCs.

\section{Mathematical model}

Assuming a plasma-RBC solution that has volume, $V$, volume fraction, $\Phi$, and number of particles, $N$.

Each particle has a volume, $V_{p}$. The permittivity and the conductivity of this solution can be modeled by the following equations [3]:

$$
\varepsilon(\omega)=\varepsilon(\infty)+\frac{\varepsilon(0)-\varepsilon(\infty)}{1+j \omega \tau}
$$

Where $\varepsilon(\infty)$ and $\varepsilon(0)$ are the dielectric constants at infinity and zero frequencies, respectively. $\tau$ is the relaxation time for the particle as function of its size, volume fraction, and the rigidity of the particle.

The volume fraction of the plasma-RBCs solution is calculated by:

$$
\Phi=\frac{N * V p}{V}
$$

\section{Equation of effective permittivity:}

The effective permittivity for the cells within the plasma can be calculated from the following eqution [10]:

$$
\varepsilon *=\varepsilon_{\mathrm{a}} *+\frac{\Phi \varepsilon_{\mathrm{a}} *}{3} \sum_{k=x, y, z} \frac{\left(\varepsilon_{\mathrm{p}} *-\varepsilon_{\mathrm{a}} *\right)}{\varepsilon_{\mathrm{a}} *+A_{k}\left(\varepsilon_{\mathrm{p}} *-\varepsilon_{\mathrm{a}} *\right)}
$$

the axial polarization factor of the cell as function of its shape [10]. $\varepsilon_{s}^{*}$ and $\varepsilon^{*}{ }_{p}$ are the equivalent permittivity of the plasma and the cell, respectively.

\section{Surface acoustic wave Equations}

Surface acoustic wave (SAW) is an acoustic wave generated and received between two transducers called interdigital transducers (IDTs). The first IDT generates the wave to the receiving one on the other side passing through the medium between them.

Depending on the electrical characteristics of this medium between them, a corresponding variation is associated with this generated wave and received by the second IDT. This variation is called the wave's perturbation, which is classified as frequency (velocity) perturbation and amplitude perturbation [11]. The related equations for the timefrequency perturbation is written as [9]:

$$
\frac{\Delta f}{f}(t)=\frac{\Delta v}{v}(t)=-\frac{K^{2}}{2} * \frac{\left(\frac{\sigma(t)}{\omega}\right)^{2}+\varepsilon_{0}\left(\varepsilon(t)-\varepsilon_{r}\right)\left(\varepsilon(t) \varepsilon_{0}+\varepsilon_{p}\right)}{\left(\frac{\sigma(t)}{\omega}\right)^{2}+\left(\varepsilon(t) \varepsilon_{0}+\varepsilon_{p}\right)^{2}}
$$

Where $\frac{\Delta v}{v}$ and $\frac{\Delta f}{f}$ are the velocity and frequency shifts, respectively, as compared to the input or the transmitted signal. $\varepsilon_{r}$ is the reference solution (the plasma-RBCs). $\omega$ is the angular frequency, $K^{2}$ is the mechanical coupling. $\varepsilon_{p}$ is the dielectric constant of the substrate and $\varepsilon_{0}$ is the dielectric constant of free space.

From this equation we can deduce that the response of this SAW sensor is a signal with shifted frequency, $\Delta f$, but this shift varies with time, since the concentration of the RBCs varies during the sedimentation.

To clarify the SAW response, during the sedimentation process two solutions will be formed in the top of the sedimentation tube, which are RBCs-plasma with subscript 1 and pure plasma solution with subscript 2 as shown in Fig.1.

As shown in Fig.1a, during the sedimentation process, RBCs will separate from the plasma and move down. So three layers will be formed, which are the plasma (top) and the RBCs-plasma (medium) with normal concentration and RBCsplasma with higher concentration (bottom), respectively.

With the progress of time, the heights of these two liquids will vary. The time rates of changing for these heights are related to the sedimentation velocity, where higher velocity means high rates of these lengths or heights. The sedimentation velocity of the RBCs as function of their radii and the volume fractions is given by [13].

$$
v=\left(\rho_{R B C}-\rho_{\text {plas }}\right) * g * d^{2} / 18 \mu(1+2.5 \Phi)
$$

$\rho_{R B C}$ and $\rho_{\text {plas }}$ are the densities of the RBCs and plasma, respectively. $d$ is the cell radius, and $\mu$ is the viscosity of the free plasma. $g$ is the acceleration of gravity.

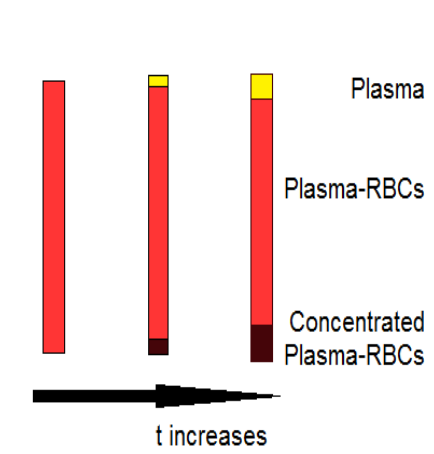

(a)

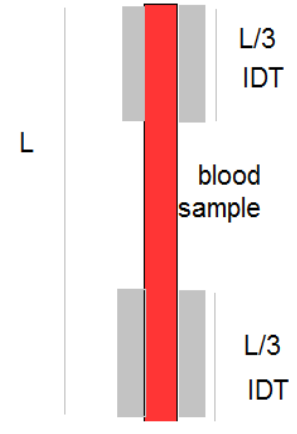

(b)
Fig.1 a): The sedimentation process and formation of three different solutions. b) SAW with blood sample.

If we consider dividing the tube blood sample into three divisions as shown in Fig.1b, the conductivity $\sigma(t)$ of the solution under the IDT can be calculated by [9]:

$$
\sigma(t)=\sigma_{1}\left(\frac{l}{L_{1}}\right)+\sigma_{2}\left(\frac{L_{1}-l}{L_{1}}\right)
$$

Also, the equation of the total dielectric constant of the solution under the IDT, $\varepsilon(t)$ is given by [9]: 


$$
\varepsilon(t)=\varepsilon_{1}\left(\frac{l}{L_{1}}\right)+\varepsilon_{2}\left(\frac{L_{1}-l}{L_{1}}\right)
$$

As applying equations 6 and 7 in equation 4, the frequency response will be deduced as function of the mechanical and the electrical characteristics of the RBCs.

So the mechanism of the SAW-bioimpedance is summarized in the following steps:

Step 1: Calculate $v$ and $l$.

Step 2: Calculate $\sigma(t)$ and $\varepsilon(t)$ (Eqs. 6 and 7).

Step 3: Calculate $\frac{\Delta v}{v}$ as a function of the frequency and the calculated parameters in step 3.

Step 4: I will increase with increasing time.

Step 5: Repeat the steps again from step 1.

Results

As mentioned before, this model deals with the mechanical and the electrical properties of the RBCs in the plasma solution. Hence, this section will discuss the relation between the ESR of the RBCs and their bioimpedances by using the SAW sensor for different cases. These cases are related to the formation of the RBCs - either the volume or the number of the RBCs. These cases are corresponding to the influence of different diseases on the blood.

\section{Relation between ESR and SAW response}

Fig. 2 shows the response of the SAW with different volume fractions. The reference or the normal value of the volume fraction is between $40 \%$ and $50 \%$ and it will be the reference case to compare the cases of the RBCs.

Changing the volume has an influence on the sedimentation rate as was shown in Eqn.5, and consequently on the value of the variation length under the sensor and so the conductivity and the permittivity of the solution as in Eqs. 6 and 7.

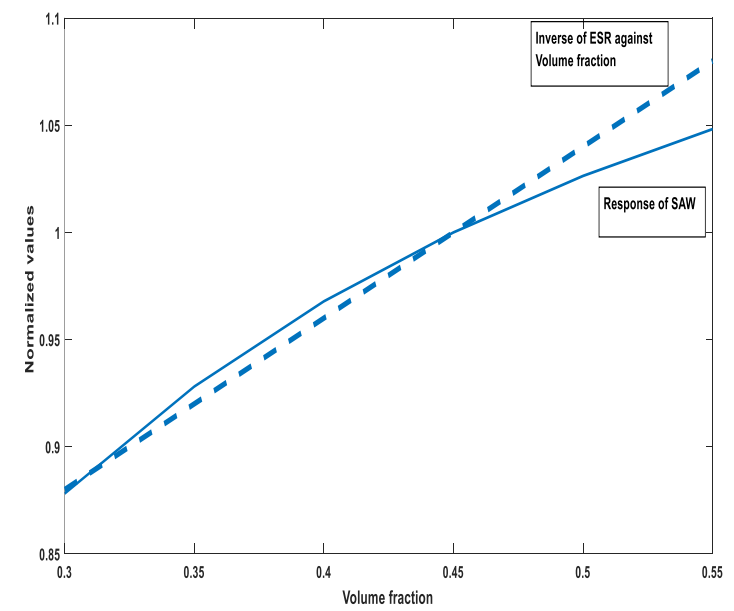

Fig.2: SAW Responses and ESR of the RBCs with different volume fractions.
RBCs with different radii (different volumes) and constant volume fractions

The variation of the volume affects directly the ESR of the RBCs with an inverse relation. This relation is shown clearly in Fig. 3 with the same start value because the same volume fraction but with different radii.

Increasing the volumes of the RBCs accelerate the rate of the sedimentation that corresponds to the transfer length, I. Increasing the volume fraction changes the rate of the length, I from one time to another (see Fig.1).

This case is equivalent to the influence some diseases have on the RBCs.

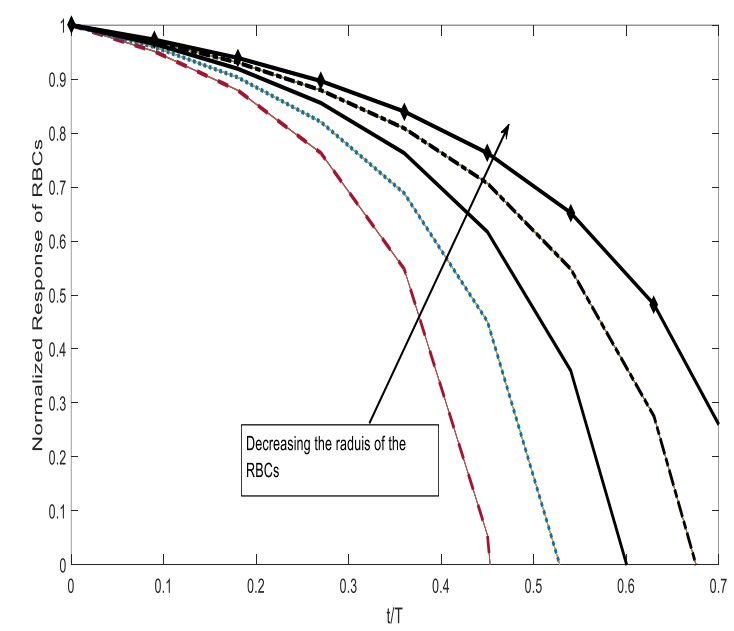

Fig.3: Response of SAW with different volume fractions.

The same volumes of the RBCs but with different volume fractions

From the modelling equations, different volume fractions means different time responses of the SAW sensor as shown in Fig.4.

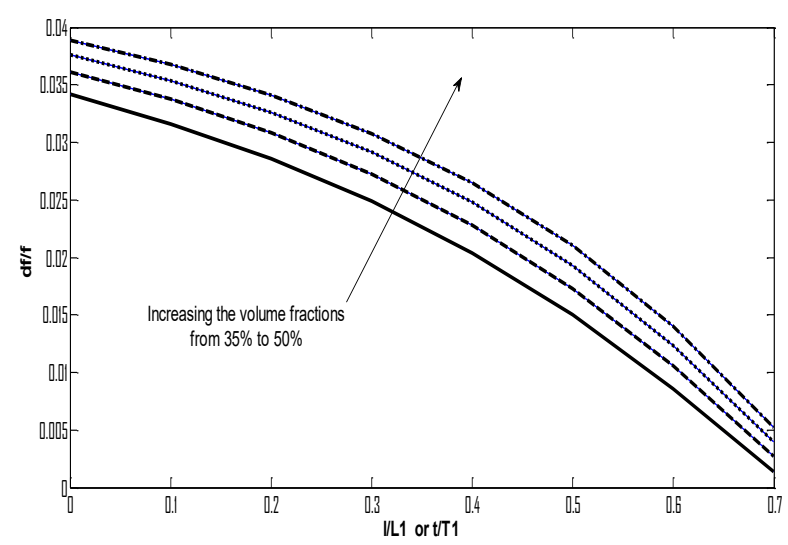

Fig.4: The responses of the RBCs for the same volumes at different volume fractions $(0.35,0.40,0.45,0.50)$

Fig.4 shows the responses of the RBCs with four different volume fractions, which are $35 \%, 40 \%, 45 \%$ and $50 \%$. All responses are shown as parallel lines, where the same volumes of the RBCs give different values of the frequency shifts, because of different volume fractions. 
Microcytes and macrocytes are practical cases corresponding to the variation of the volumes of the RBCs that correspond to some cases like e.g. influence of alcohol.

Fig. 5 shows the responses of the SAW for the microcytes and macrocytes as compared to the normal RBCs.

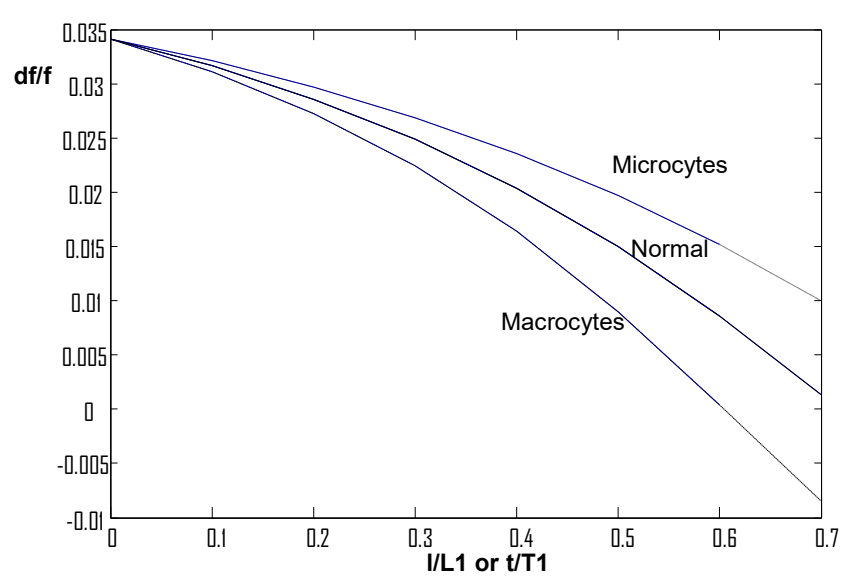

Fig.5: Responses of microcytes and macrocytes as compared to the normal volume of RBCs.

\section{Response of oblate cells}

Oblate cells are red blood cells inherent to some pathological cases. Detecting this kind of cells depends on a different feature called polarization factor as function of the dimensions and the form of the cell $[10,12]$.

The simplest cell's form is the spherical cells so it is considered as the reference case for calculating the other electrical parameters as referred to the parameters of the spherical cells [9].

The estimated electrical values of the oblate cells (from [9]) will be applied to this model to study the response of these cells. Fig. 6 shows the electrical response of the SAW corresponding to the electrical parameters of the oblate cells for three different volume fractions $(0.3,0.4$, and 0.5$)$ referring to the spherical cases with the same volume fractions.

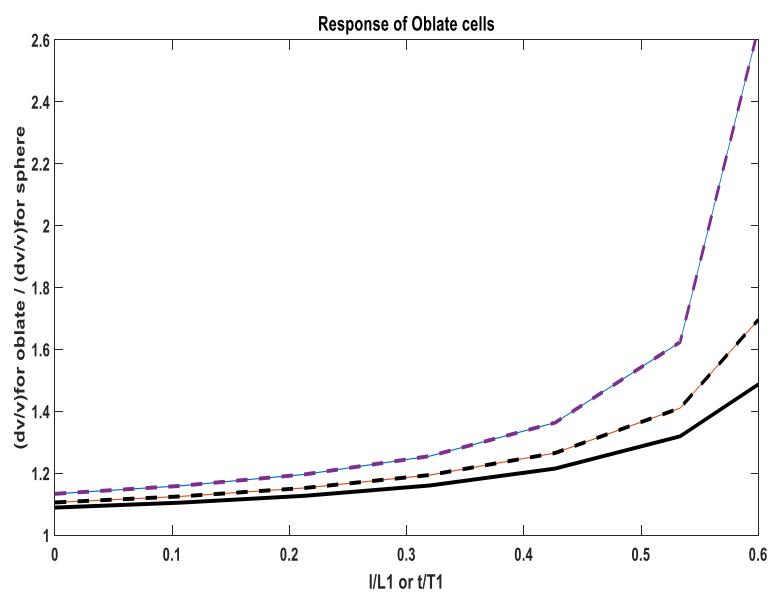

Fig.6: Response of oblate cells as compared to the spherical cells with the same concentrations.

\section{Discussion}

Depending on the physical characteristics of the RBCs and the sensitivity of the surface acoustic waves, a new model has been proposed. This model shows its ability with suitable sensitivity for detecting the variation in the RBCs comparing to the normal RBCs. These variations are the volume fractions, the volumes, and different kinds of RBCs. The applications may be extended to procedure a complete blood analysis.

\section{References}

1. Grimnes S., and Martinsen $\varnothing$.G. Bioimpedance and bioelectricity basic, $2^{\text {nd }}$ ed. Academic Press, New York, 2000.

2. Asami K., and Yonezawa T. Dielectric behavior of wild-type yeast and vacuole-deficient mutant over a frequency range of $10 \mathrm{kHz}$ to $10 \mathrm{GHz}$. Biophysical Journal. 71(4); 2192-2200: 1996. https://dx.doi.org/10.1016/\$0006-3495(96)79420-1

3. Martinsen $\varnothing$.G., Grimnes S., and Schwan H.P. Interface Phenomena and dielectric properties of biological tissue. Encyclopedia of Surface and Colloid Science. 2643-2652: 2002.

4. Pradhan R., Mitra A., and Das S. Impedimetric characterization of human blood using three-electrode based ECIS devices. J. Electr. Bioimp. 3; 12-19: 2012. https://doi.org/10.5617/jeb.238

5. Hossain Q.D., and Dhar S.K. Characterization of Physiological Glucose Concentration Using Electrical Impedance Spectroscopy. IJCSI International Journal of Computer Science. 10(1); 105-111: 2013.

6. Ni A., Cheema T.A., and Park C.W. Numerical Study of RBC Motion and Deformation through Microcapillary in Alcohol Plasma Solution. Open Journal of Fluid Dynamics. 5(1); 26-33: 2015. https://doi.org/10.4236/ojfd.2015.51004

7. Bbosa G.S., Kyegombe D.B., Anokbonggo W.W., Lubega A., Mugisha A., and Ogwal-Okeng J. Effect of chronic alcohol consumption on the red blood cell count and RBC indices in the HIV infected patients on d4T/3TC/NVP drug regimen in Uganda. International Journal of Basic \& Clinical Pharmacology. 2(5); 528536: 2013. https://doi.org/10.5455/2319-2003.ijbcp20131004

8. Punter-Villagrasa J., Cid J., Páez-Avilés C., Rodríguez-Villarreal I., Juanola-Feliu E., Colomer-Farrarons J., and Miribel-Català P.L. An Instantaneous Low-Cost Point-of-Care Anemia Detection Device. Sensors. 15: 4564-4577: 2015. https://doi.org/10.3390/s150204564

9. EL-Dosoky M.A.A. New Proposed Algorithm for Determining the Electrical Characteristics of the Prolates and Oblates RBCs. International Journal of Computer Applications. 131(6); 6-9: 2015. https://doi.org/10.5120/ijca2015907436

10. Raicu V., Saibara T., Enzan H., and Irimajiri A. Dielectric properties of rat liver in vivo: analysis by algorithm hepatocytes in the tissue architecture. Bioelectrochemistry and Bioenergetics. 47(2); 333342: 1998. https://doi.org/10.1016/S0302-4598(98)00172-X

11. El Zarie S.T., and EL-Dosoky M.A.A. Diagnosis of Blood Diseases Using Shear Horizontal Surface Acoustic Waves. $30^{\text {th }}$ National Radio Science Conference (NRSC 2013), National Telecommunication Institute, Egypt, April 16-18, 2013. https://doi.org/10.1109/NRSC.2013.6587960 